International Journal of Algebra, Vol. 8, 2014, no. 4, 159 - 174

HIKARI Ltd, www.m-hikari.com

http://dx.doi.org/10.12988/ija.2014.312135

\title{
On the Gorenstein Property of the Fiber Cone to Filtration
}

\author{
P. H. Lima ${ }^{1}$ and V. H. Jorge Pérez ${ }^{2}$
}

Copyright (c) 2014 P. H. Lima and V. H. Jorge Pérez. This is an open access article distributed under the Creative Commons Attribution License, which permits unrestricted use, distribution, and reproduction in any medium, provided the original work is properly cited.

\begin{abstract}
Let $(A, \mathfrak{m})$ be a Noetherian local ring and $\mathfrak{F}=\left(I_{n}\right)_{n \geq 0}$ a filtration. In this paper, we study the Gorenstein properties of the fiber cone $F(\mathfrak{F})$, where $\mathfrak{F}$ is a Hilbert filtration. Suppose that $F(\mathfrak{F})$ and $G(\mathfrak{F})$ are CohenMacaulay. If in addition, the associated graded ring $G(\mathfrak{F})$ is Gorenstein; similarly to the $I$-adic case, we obtain a necessary and sufficient condition, in terms of lengths and minimal number of generators of ideals, for Gorensteiness of the fiber cone. Moreover, we find a description of the canonical module of $F(\mathfrak{F})$ and show that even in the Hilbert filtration case, the multiplicity of the canonical module of the fiber cone is upper bounded by multiplicity of the canonical modules of the associated graded ring.
\end{abstract}

Keywords: fiber cone, associated graded ring, good and Hilbert filtration, reduction number

\section{Introduction}

Let $(A, \mathfrak{m})$ be a Noetherian local ring and $A \supset I \supset I^{2} \supset \ldots$ the adicfiltration. Then we have important graded algebras such as $R(I):=\oplus_{n \geq 0} I^{n} t^{n}$, the associated graded ring $G(I):=\oplus_{n \geq 0} I^{n} / I^{n+1}$ and the fiber cone $F(I):=$ $\oplus_{n \geq 0} I^{n} / \mathfrak{m} I^{n}=R(I) / \mathfrak{m} R(I)$. These algebras may be naturally generalized for

\footnotetext{
${ }^{1}$ Work partially supported by CNPq-Brazil 141973/2010-2 and by Capes-Brazil.

${ }^{2}$ Work supported by CNPq-Brazil - Grant 309033/2009-8, Procad-190/2007.
} 
the general filtration case of ideals, that is, $\mathfrak{F}: A \supset I_{1} \supset I_{2} \supset \ldots$. We may ask when these algebras are Gorenstein or Cohen-Macaulay and other properties. This answer is given in many papers ([14], [7], [16], [26], [17], [11], [12], [20]). In [14], it is determined, under certain classes of ideals, the exponent $n \geq 0$ such that $R\left(I^{n}\right)$ and $G\left(I^{n}\right)$ are Gorenstein. Goto and Nishida [7] investigate the Cohen-Macaulay and Gorenstein properties of symbolic Rees algebras for one-dimensional prime ideals in Cohen-Macaulay local rings, and then they generalize to Rees algebras $R(\mathfrak{F})$ and graded rings $G(\mathfrak{F})$ associated to general filtrations of ideals in arbitrary Noetherian local rings. Moreover, in [16], Zarzuela and Hoa give some consequences which generalize well known results in the $I$-adic case. For instance, they show that, under certain assumptions, the Gorensteinness of $R(\mathfrak{F})$ implies the Gorensteinness of $G(\mathfrak{F})$. Trung, Viêt and Zarzuela, in [26], extend the Ikeda's result [17] to Rees algebra of filtrations. In the article [11], Heinzer, Kim and Ulrich give a criterion, in terms of ideals, for the Gorensteinness of $G(I)$. Next they generalized this result for Hilbert filtrations [12]. Jayanthan, Puthenpurakal and Verma, in [20], study the Gorenstein property of $F(I)$ of $\mathfrak{m}$-primary ideals and the ones of almost minimal multiplicity.

In this article, we are interested on the Gorenstein property of the fiber cone to filtration. In a recent article, for the $I$-adic case, Jayanthan and Nanduri [19], find descriptions, in terms of ideals, for the canonical module of the fiber cone. They also show that if both the associated graded ring and fiber cone are Cohen-Macaulay, then the regularities of the their canonical modules are equal. Furthermore, when $F(I)$ is Cohen-Macaulay and $G(I)$ is Gorenstein, they give a criterion to say when the fiber cone is Gorenstein. The main goal in this paper is to give an analogous theory on the Gorenstein property of the fiber cone for general filtration. We show that the results obtained in [19] may be generalized for the Hilbert filtration.

In this paper we extend the Theorem 15 and Theorem 33 in [4] for the good filtration case. Moreover, we find for the Hilbert filtration case, Gorenstein properties of the fiber cone and descriptions of its canonical module in terms of ideals. We also see that the multiplicity of the canonical module of the fiber cone is less than or equal to the multiplicity of the canonical module of the associated graded ring even for Hilbert filtration and that if in addition, $G(\mathfrak{F})$ is Gorenstein, the equality is true if only if $I_{1}=\mathfrak{m}$. The main results in this section generalize the results in the section 3 of [19] to Hilbert filtration. 


\section{Gorenstein Property of the Fiber Cone to Filtrations}

Throughout this section, $(A, \mathfrak{m})$ is a Noetherian local ring of dimension $d$ with an infinite residue field and $\mathfrak{F}$ is a good filtration. Moreover we always suppose that the fiber cone $F(\mathfrak{F})$ and the associated graded ring $G(\mathfrak{F})$ are CohenMacaulay. If the dimension of the fiber cone is greater than zero, by [4, Lemma 3.1], $I_{n+1} \subseteq \mathfrak{m} I_{n}$ for all $n \geq 0$ and then $F(\mathfrak{F})=G(\mathfrak{F}) / \mathfrak{m} G(\mathfrak{F})$. In this article we assume this fact if $A$, and therefore $F(\mathfrak{F})$, has dimension zero. It is required in the most of the results in this article such as the Proposition below. In [19], Jayanthan and Nanduri obtain, for the $I$-adic filtration case, an expression for the canonical module of the fiber cone. We verify this result remains true for the case of a Hilbert filtration $\mathfrak{F}$.

Proposition 2.1. Let $(A, \mathfrak{m})$ be a Noetherian local ring and $\mathfrak{F}$ a Hilbert filtration such that the associated graded ring $G(\mathfrak{F})$ is Cohen-Macaulay. Let $\omega_{G(\mathfrak{F})}=\oplus_{n \in \mathbb{Z}} \omega_{n}$ and $\omega_{F(\mathfrak{F})}$ be the canonical modules of $G(\mathfrak{F})$ and $F(\mathfrak{F})$ respectively. Then

(1) $\omega_{F(\mathfrak{F})} \cong \oplus_{n \in \mathbb{Z}}\left(0: \omega_{n} \mathfrak{m}\right)$;

(2) $a(F(\mathfrak{F}))=a(G(\mathfrak{F}))=r_{J}(\mathfrak{F})-d$, where $r:=r_{J}(\mathfrak{F})$ is the reduction number of $\mathfrak{F}$ with respect to $J$ to any reduction minimal $J \subseteq I_{1}$;

(3) $a\left(F\left(\mathfrak{F}^{(k)}\right)\right)=\left[\frac{a(F(\mathfrak{F}))}{k}\right]=\left[\frac{r-d}{k}\right]$ for any $k \in \mathbb{N}$.

(4) if $G(\mathfrak{F})$ is Gorenstein,

$$
\omega_{F(\mathfrak{F})} \cong \bigoplus_{n \in \mathbb{Z}} \frac{\left(I_{n+r-d+1}: \mathfrak{m}\right) \cap I_{n+r-d}}{I_{n+r-d+1}} .
$$

Proof. Firstly, note by [16, Proposition 3.6 and Theorem 3.8] that the $a$ invariants of $G(\mathfrak{F})$, defined with support $G(\mathfrak{F})_{+}$and $\mathfrak{M}$, are equal.

(1) By $\left[1\right.$, Theorem 4.5.6], one has $\operatorname{dim} G(\mathfrak{F})=d$. Since $I_{1}$ is an $\mathfrak{m}$-primary ideal, $\operatorname{dim} F\left(I_{1}\right)=d$. By [16, Lemma 2.8], $\operatorname{dim} F(\mathfrak{F})=\operatorname{dim} F\left(I_{1}\right)$. Thus $\operatorname{dim} G(\mathfrak{F})=\operatorname{dim} F(\mathfrak{F})=d$. By hypothesis, $G(\mathfrak{F})$ is Cohen-Macaulay, so by using [9, Corollary 36.14], we have

$$
\omega_{F(\mathfrak{F})} \cong \operatorname{Hom}_{G(\mathfrak{F})}\left(F(\mathfrak{F}), \omega_{G(\mathfrak{F})}\right) .
$$

It is easy to show that

$$
\operatorname{Hom}_{G(\mathfrak{F})}\left(F(\mathfrak{F}), \omega_{G(\mathfrak{F})}\right) \cong\left(0: \omega_{G(\mathfrak{F})} \mathfrak{m} G(\mathfrak{F})\right)=\left(0: \omega_{G(\mathfrak{F})} \mathfrak{m}\right)=\oplus_{n \in \mathbb{Z}}\left(0:_{\omega_{n}} \mathfrak{m}\right)
$$

The result follows. 
(2) By $\left[1\right.$, Definition 3.6.13], $a(F(\mathfrak{F}))=-\min \left\{n \mid\left[\omega_{F(\mathfrak{F})}\right]_{n} \neq 0\right\}$. Since $A / I_{1}$ is Artinian and $\omega_{n}$ is a finite $A / I_{1}$-module, it follows that $\omega_{n}$ is Artinian. By [18, Theorem A.33], $\omega_{n}$ is a $\mathfrak{m} / I_{1}$ - torsion and by [18, Example A.9],

$$
\left(0: \omega_{n} \mathfrak{m}\right)=\left(0: \omega_{n} \mathfrak{m} / I_{1}\right) \subseteq \omega_{n}
$$

is an essential extension. Thus

$$
\left(0: \omega_{n} \mathfrak{m}\right) \neq 0 \text { if and only if } \omega_{n} \neq 0 .
$$

Therefore by $(\mathbf{1}), a(F(\mathfrak{F}))=a(G(\mathfrak{F}))$.

Through [1, Theorem 4.5.7], one has $A$ is Cohen-Macaulay. Note also that $\mathfrak{F}$ is equimultiple since $I_{1}$ is $\mathfrak{m}$-primary. As grade $G(\mathfrak{F})_{+}=d$, the hypothesis of [16, Proposition 3.6] are satisfied and then the second equality follows.

(3) By [16, Corollary 4.6], $G\left(\mathfrak{F}^{(k)}\right)$ is Cohen-Macaulay. By [16, Theorem 4.4], $a\left(G\left(\mathfrak{F}^{(k)}\right)\right)=\left[\frac{a(G(\mathfrak{F}))}{k}\right]$. Applying (2) to Veronesean filtration $\mathfrak{F}^{(k)}$, we have

$$
a\left(F\left(\mathfrak{F}^{(k)}\right)\right)=a\left(G\left(\mathfrak{F}^{(k)}\right)\right)=\left[\frac{a(G(\mathfrak{F}))}{k}\right]=\left[\frac{a(F(\mathfrak{F}))}{k}\right]=\left[\frac{r-d}{k}\right] .
$$

(4) Let suppose that $G(\mathfrak{F})$ is Gorenstein. By $[9,36.13]$,

$$
\omega_{G(\mathfrak{F})} \cong G(\mathfrak{F})(a(G(\mathfrak{F})))=G(\mathfrak{F})(r-d) .
$$

That is $\omega_{n}=I_{n+r-d} / I_{n+r-d+1}$ for any $n$. By using the last fact and (1), we have

$$
\begin{aligned}
\omega_{F(\mathfrak{F})} \cong \oplus_{n \in \mathbb{Z}}\left(0: \omega_{n} \mathfrak{m}\right) & =\bigoplus_{n \in \mathbb{Z}} \frac{\left(I_{n+r-d+1}: \mathfrak{m}\right) \cap I_{n+r-d}}{I_{n+r-d+1}} \\
& \left(=\bigoplus_{n \geq d-r} \frac{\left(I_{n+r-d+1}: \mathfrak{m}\right) \cap I_{n+r-d}}{I_{n+r-d+1}}\right) .
\end{aligned}
$$

Corollary 2.2. Suppose that $A$ has dimension $\geq 2$. If $R(\mathfrak{F})$ is Gorenstein,

$$
\omega_{F(\mathfrak{F})} \cong \bigoplus_{n \in \mathbb{Z}}\left(0: \omega_{n} \mathfrak{m}\right)=\bigoplus_{n \in \mathbb{Z}} \frac{\left(I_{n-1}: \mathfrak{m}\right) \cap I_{n-2}}{I_{n-1}}
$$

That is, the canonical module $\omega_{F(\mathfrak{F})}$ does not depend on the dimension $d$ and the number reduction $r$.

Proof. Use [16, Corollary 3.11].

The Corollary below may be also view as a particular case of $[19$, Theorem $2.3]$. 
Corollary 2.3. Let $\mathfrak{F}$ be an equimultiple good filtration. If $G(\mathfrak{F})$ is CohenMacaulay then $\operatorname{reg} G(\mathfrak{F}) \leq \operatorname{reg} F(\mathfrak{F})$. If in addition $F(\mathfrak{F})$ is Cohen-Macaulay, then $\operatorname{reg} G(\mathfrak{F})=r=\operatorname{reg} F(\mathfrak{F})$.

Proof. Use [23, Theorem 4.6] and [16, Proposition 3.6].

If the rings $G(\mathfrak{F})$ and $F(\mathfrak{F})$ are Cohen-Macaulay, we see that for any Hilbert filtration, the regularities of $\omega_{G(\mathfrak{F})}$ and $\omega_{F(\mathfrak{F})}$ are equal. If in addition either $G(\mathfrak{F})$ or $F(\mathfrak{F})$ is Gorenstein, the regularity of their canonical modules are equal to the dimension of the base ring.

Corollary 2.4. If the rings $G(\mathfrak{F})$ and $F(\mathfrak{F})$ are Cohen-Macaulay, one has $\operatorname{reg} \omega_{G(\mathfrak{F})}=\operatorname{reg} \omega_{F(\mathfrak{F})}$. Furthermore, if either $G(\mathfrak{F})$ or $F(\mathfrak{F})$ is Gorenstein, $\operatorname{reg} \omega_{G(\mathfrak{F})}=\operatorname{reg} \omega_{F(\mathfrak{F})}=d$.

Proof. The case $d=0$ follows of some arguments in the proof for positive dimension. Assume $d>0$. By [22, Proposition 2.2] and hypothesis, we get a minimal reduction $J$ of $\mathfrak{F}$ minimally generated by $x_{1}, \ldots, x_{d}$ such that $x_{1}^{*}, \ldots, x_{d}^{*} \in G(\mathfrak{F})$ and $x_{1}^{o}, \ldots, x_{d}^{o} \in F(\mathfrak{F})$ are regular sequences. Then

$F(\mathfrak{F}) /\left(x_{1}^{o}, \ldots, x_{d}^{o}\right) \cong F\left(\mathfrak{F} /\left(x_{1}, \ldots, x_{d}\right)\right)$ and $G(\mathfrak{F}) /\left(x_{1}^{*}, \ldots, x_{d}^{*}\right) \cong G\left(\mathfrak{F} /\left(x_{1}, \ldots, x_{d}\right)\right)$

Then by [1, Corollary 3.6.14],

$$
\operatorname{reg} \omega_{F(\mathfrak{F})}=\operatorname{reg}\left(\omega_{F(\mathfrak{F})} / J^{o} \omega_{F(\mathfrak{F})}\right)=\operatorname{reg} \omega_{F(\mathfrak{F} / J)}+d
$$

and the same for $G(\mathfrak{F})$, that is, $\operatorname{reg} \omega_{G(\mathfrak{F})}=\operatorname{reg} \omega_{G(\mathfrak{F} / J)}+d$.

Since $\operatorname{dim} F(\mathfrak{F} / J)=0=\operatorname{dim} G(\mathfrak{F} / J), \operatorname{reg} \omega_{F(\mathfrak{F} / J)}=a\left(\omega_{F(\mathfrak{F} / J)}\right)$ and $\operatorname{reg} \omega_{G(\mathfrak{F} / J)}=$ $a\left(\omega_{G(\mathfrak{F} / J)}\right)$.

By applying the Proposition 2.1(1) to $\mathfrak{F} / J$, we have

$$
\omega_{F(\mathfrak{F} / J)} \cong \oplus_{n \in \mathbb{Z}}\left(0:\left[\omega_{G(\mathfrak{F} / J)}\right]_{n} \mathfrak{m}\right) .
$$

Then by $(2.1)$, we have $\left[\omega_{F(\mathfrak{F} / J)}\right]_{n} \neq 0 \Leftrightarrow\left[\omega_{G(\mathfrak{F} / J)}\right]_{n} \neq 0$ for all $n$. Hence $a\left(\omega_{F(\mathfrak{F} / J)}\right)=a\left(\omega_{G(\mathfrak{F} / J)}\right)$ so that $\operatorname{reg} \omega_{G(\mathfrak{F})}=\operatorname{reg} \omega_{F(\mathfrak{F})}$.

Furthermore, if for example $G(\mathfrak{F})$ is Gorenstein, by [1, Proposition 3.6.11], $\omega_{G(\mathfrak{F})} \cong G(\mathfrak{F})(r-d)$. Then

$$
\operatorname{reg} \omega_{G(\mathfrak{F})}=\operatorname{reg} G(\mathfrak{F})(r-d)=\operatorname{reg} G(\mathfrak{F})-r+d
$$

Since $G(\mathfrak{F})$ is Cohen-Macaulay, by [16, Proposition 3.6], reg $G(\mathfrak{F})=r$. Hence we get $\operatorname{reg} \omega_{G(\mathfrak{F})}=d$. The result follows similarly if we suppose that $F(\mathfrak{F})$ is Gorenstein. 
In the Corollary below we see other descriptions (in terms of quotient ideals) of the canonical module of the fiber cone and the canonical module of the associated graded ring.

Corollary 2.5. Let $(A, \mathfrak{m})$ be a Gorenstein Noetherian local ring such that $d>0$ and $\mathfrak{F}$ a Hilbert filtration. Suppose that $G(\mathfrak{F})$ is Cohen-Macaulay. Then

$$
\omega_{G(\mathfrak{F})}=\bigoplus_{n \in \mathbb{Z}} \frac{\left(J^{n+r-d}: I_{r}\right)}{\left(J^{n+r-d+1}: I_{r}\right)}
$$

and

$$
\omega_{F(\mathfrak{F})}=\bigoplus_{n \in \mathbb{Z}} \frac{\left(J^{n+r-d+1}: \mathfrak{m} I_{r}\right) \cap\left(J^{n+r-d}: I_{r}\right)}{\left(J^{n+r-d+1}: I_{r}\right)} .
$$

Furthermore, $F(\mathfrak{F})$ is Gorenstein if and only if

$$
\frac{\left(J^{n+1}: \mathfrak{m} I_{r}\right) \cap\left(J^{n}: I_{r}\right)}{\left(J^{n+1}: I_{r}\right)} \cong \frac{I_{n}}{\mathfrak{m} I_{n}}
$$

for $n \geq 0$.

Proof. Due to hypothesis in this Corollary, the hypothesis from [12] are also satisfied and then

$$
\omega_{R^{\prime}(\mathfrak{F})}=\bigoplus_{n \in \mathbb{Z}}\left(J^{n+r}: I_{r}\right) t^{n+d-1} .
$$

By [1, Corollary 3.6.14],

$$
\omega_{G(\mathfrak{F})}=\omega_{R^{\prime}(\mathfrak{F}) /\left(t^{-1}\right) R^{\prime}(\mathfrak{F})} \cong\left(\omega_{R^{\prime}(\mathfrak{F})} / t^{-1} \omega_{R^{\prime}(\mathfrak{F})}\right)(-1) .
$$

Hence $\omega_{G(\mathfrak{F})}=\bigoplus_{n \in \mathbb{Z}} \frac{\left(J^{n+r-d}: I_{r}\right)}{\left(J^{n+r-d+1}: I_{r}\right)}$.

Next we use the Proposition 2.1(1) to get

$$
\begin{aligned}
\omega_{G(\mathfrak{F})} & =\bigoplus_{n \in \mathbb{Z}}\left(0:\left[\omega_{G(\mathfrak{F})]_{n}} \mathfrak{m}\right)\right. \\
& =\bigoplus_{n \in \mathbb{Z}} \frac{\left(J^{n+r-d+1}: \mathfrak{m} I_{r}\right) \cap\left(J^{n+r-d}: I_{r}\right)}{\left(J^{n+r-d+1}: I_{r}\right)}
\end{aligned}
$$

Now suppose $G(\mathfrak{F})$ is Gorenstein. Then

$$
\omega_{F(\mathfrak{F})} \cong F(\mathfrak{F})(r-d)=\bigoplus_{n \in \mathbb{Z}} \frac{I_{n+r-d}}{\mathfrak{m} I_{n+r-d}}
$$

Comparing both the above graded isomorphism, we have

$$
\frac{\left(J^{n+1}: \mathfrak{m} I_{r}\right) \cap\left(J^{n}: I_{r}\right)}{\left(J^{n+1}: I_{r}\right)} \cong \frac{I_{n}}{\mathfrak{m} I_{n}},
$$

for $n \geq 0$. The converse follows easily. 
Lemma 2.6. Suppose $\ell=\operatorname{dim} F(\mathfrak{F})=1$ and let $J=(a)$ be a minimal reduction of a good filtration $\mathfrak{F}$. Assume that $I_{1}$ contains a regular element. Then for all $n$ and $0 \leq i \leq n-1$, we have

$$
\lambda\left(I_{n} /\left(\mathfrak{m} I_{n}+a^{n-i} I_{i}\right)\right)=\mu\left(I_{n}\right)-\mu\left(I_{i}\right)+\lambda\left(\left(a^{n-i} I_{i} \cap \mathfrak{m} I_{n}\right) / a^{n-i} \mathfrak{m} I_{i}\right) .
$$

Particularly,

$$
\lambda\left(I_{n} /\left(\mathfrak{m} I_{n}+a I_{n-1}\right)\right)=\mu\left(I_{n}\right)-\mu\left(I_{n-1}\right)+\lambda\left(\left(a I_{n-1} \cap \mathfrak{m} I_{n}\right) / a \mathfrak{m} I_{n-1}\right) .
$$

Proof. The assumption on $I_{1}$ gives that $a$ is regular. The exact sequence

$$
0 \rightarrow\left(a^{n}\right) /\left(\mathfrak{m} I_{n} \cap\left(a^{n}\right)\right) \rightarrow I_{n} / \mathfrak{m} I_{n} \quad \rightarrow \quad I_{n} /\left(\mathfrak{m} I_{n}+\left(a^{n}\right)\right) \rightarrow 0
$$

and the equality $\mathfrak{m} I_{n} \cap\left(a^{n}\right)=a^{n} \mathfrak{m}(J$ is analytically independent in $\mathfrak{F})$ gives $\lambda\left(I_{n} /\left(\mathfrak{m} I_{n}+\left(a^{n}\right)\right)\right)=\mu\left(I_{n}\right)-\mu\left(J^{n}\right)=\mu\left(I_{n}\right)-1$. For $1 \leq i \leq n-1$, we have the following exact sequences

$$
\begin{aligned}
0 & \rightarrow a^{n-i} I_{i} /\left(a^{n-i} I_{i} \cap \mathfrak{m} I_{n}\right) \rightarrow I_{n} / \mathfrak{m} I_{n} \rightarrow I_{n} /\left(\mathfrak{m} I_{n}+a^{n-i} I_{i}\right) \rightarrow 0 \\
0 & \rightarrow\left(a^{n-i} I_{i} \cap \mathfrak{m} I_{n}\right) / a^{n-i} \mathfrak{m} I_{i} \rightarrow a^{n-i} I_{i} / a^{n-i} \mathfrak{m} I_{i} \rightarrow a^{n-i} I_{i} /\left(a^{n-i} I_{i} \cap \mathfrak{m} I_{n}\right) \rightarrow 0 .
\end{aligned}
$$

We also have the isomorphism

$$
a^{n-i} I_{i} / a^{n-i} \mathfrak{m} I_{i} \cong I_{i} / \mathfrak{m} I_{i}
$$

since $a$ is a regular element. The result follows due to the additivity of $\lambda$.

Notation 2.7. If $a_{1}, \ldots, a_{k} \in A$ and $\mathfrak{F}$ is a good filtration, we denote by $I_{n}^{(k)}=$ $\left(I_{n}+\left(a_{1}, \ldots, a_{k}\right)\right) /\left(a_{1}, \ldots, a_{k}\right)$ the $n$-th term of the filtration $\mathfrak{F} /\left(a_{1}, \ldots, a_{k}\right)$ and the quotient ring $A /\left(a_{1}, \ldots, a_{k-1}\right)$ by $A_{k-1}$.

Lemma 2.8. If $a_{1}, \ldots, a_{k} \in I_{1}$ are such that $a_{1}^{*}, \ldots, a_{k}^{*} \in G(\mathfrak{F})$ and $a_{1}^{o}, \ldots, a_{k}^{o} \in$ $F(\mathfrak{F})$ are regular sequence, then

$$
\mu\left(I_{n}^{(k)}\right)=\sum_{i=0}^{k}(-1)^{i}\left(\begin{array}{l}
k \\
i
\end{array}\right) \mu\left(I_{n-i}\right) .
$$

Proof. The proof is done by using induction on $k$. It is easy to obtain $\left(a_{1}\right) \cap I_{n}=$ $a_{1} I_{n-1}$ for all $n \geq 0$. If $k=1$, by the isomorphism theorem,

$$
\mu\left(I_{n}^{(1)}\right)=\lambda\left(\left(I_{n}+\left(a_{1}\right)\right) /\left(\mathfrak{m} I_{n}+\left(a_{1}\right)\right)=\lambda\left(I_{n} /\left(\mathfrak{m} I_{n}+a_{1} I_{n-1}\right)\right) .\right.
$$

Let consider the following natural exact sequence

$$
0 \rightarrow a_{1} I_{n-1} /\left(a_{1} I_{n-1} \cap \mathfrak{m} I_{n}\right) \rightarrow I_{n} / \mathfrak{m} I_{n} \rightarrow I_{n} /\left(\mathfrak{m} I_{n}+a_{1} I_{n-1}\right) \rightarrow 0
$$


From [4, Proposition 2.3(iii) and Theorem 2.8], we have $a_{1} I_{n-1} \cap \mathfrak{m} I_{n}=$ $a_{1} \mathfrak{m} I_{n-1}$. Moreover by [13, Proposition 3.5], $a_{1}$ is a regular element. Then $a_{1} I_{n-1} / a_{1} \mathfrak{m} I_{n-1} \cong I_{n-1} / \mathfrak{m} I_{n-1}$. Thus by exact sequence above, $\mu\left(I_{n}^{(1)}\right)=$ $\mu\left(I_{n}\right)-\mu\left(I_{n-1}\right)$.

Now suppose $k>1$. Hence $\bar{a}_{k} \in A_{k-1},\left(\bar{a}_{k}\right)^{*} \in G\left(\mathfrak{F} /\left(a_{1}, \ldots, a_{k-1}\right)\right)$ and $\left(\bar{a}_{k}\right)^{o} \in F\left(\mathfrak{F} /\left(a_{1}, \ldots, a_{k-1}\right)\right)$ are regular elements. We know that the equality $\mu\left(I_{n}^{(k)}\right)=\mu\left(I_{n}^{(k-1)}\right)-\mu\left(I_{n-1}^{(k-1)}\right)$ is true for $k=1$. Then, by induction on $k$,

$$
\begin{aligned}
\mu\left(I_{n}^{(k)}\right) & =\sum_{i=0}^{k-1}(-1)^{i}\left(\begin{array}{c}
k-1 \\
i
\end{array}\right) \mu\left(I_{n-i}\right)-\sum_{i=0}^{k-1}(-1)^{i}\left(\begin{array}{c}
k-1 \\
i
\end{array}\right) \mu\left(I_{n-1-i}\right) \\
& =\sum_{i=0}^{k}(-1)^{i}\left(\left(\begin{array}{c}
k-1 \\
i
\end{array}\right)+\left(\begin{array}{c}
k-1 \\
i-1
\end{array}\right)\right) \mu\left(I_{n-i}\right) \\
& =\sum_{i=0}^{k}(-1)^{i}\left(\begin{array}{c}
k \\
i
\end{array}\right) \mu\left(I_{n-i}\right)
\end{aligned}
$$

The next lemma gives several characterizations of the Cohen-Macaulay property of the fiber cone of dimension one to good filtration.

Lemma 2.9. Let $(A, \mathfrak{m})$ be a Noetherian Local ring and let $\mathfrak{F}$ a good filtration such that $I_{1}$ contains a regular element, $\operatorname{dim} F(\mathfrak{F})=1$ and $r$ is its reduction number. Then we have the following equivalent conditions:

(1) $F(\mathfrak{F})$ is Cohen-Macaulay;

(2) $\left(0:_{F(\mathfrak{F})} a^{o}\right)=0$ for any $J=(a) \subseteq I_{1}$ minimal reduction of $\mathfrak{F}$;

(3) For any minimal reduction $(a)$ of $\mathfrak{F}, I_{n} \cap\left(\mathfrak{m} I_{n+1}: a\right)=\mathfrak{m} I_{n}, 1 \leq n \leq r-1$;

(4) For any minimal reduction (a) of $\mathfrak{F}, a I_{n} \cap \mathfrak{m} I_{n+1}=a \mathfrak{m} I_{n}, 1 \leq n \leq r-1$;

(5) For any minimal reduction (a) of $\mathfrak{F}, \lambda\left(I_{n} /\left(\mathfrak{m} I_{n}+a I_{n-1}\right)\right)=\mu\left(I_{n}\right)-\mu\left(I_{n-1}\right)$, $1 \leq n \leq r$.

Proof. The equivalences (1) $\Leftrightarrow(2),(2) \Leftrightarrow(3)$ are easy to be found. The sentences (3) and (4) follow by using the fact that $a$ is regular in $A$, while (4) $\Leftrightarrow(5)$ is provided by Lemma 2.6 .

Lemma 2.10. Let $(R, \mathfrak{m})$ be a Noetherian local ring and $\mathfrak{F}$ a good filtration. Suppose $J=\left(a_{1} \ldots, a_{d}\right) R$ is such that $a_{1}, \ldots, a_{d}$ are part of a minimal generating set for $I_{1}$ and $a_{1}^{*} \ldots, a_{d-1}^{*}$ is a $G(\mathfrak{F})$-regular sequence. If $J I_{n}+\left(a_{1}, \ldots a_{i}\right) R=$ $I_{n+1}+\left(a_{1}, \ldots a_{i}\right) R$ for $1 \leq i \leq d-1$, then $J I_{n}=I_{n+1}$.

Proof. By using [16, Lemma 3.4] and induction on $i$, it is enough to prove the case $i=1$. If $J I_{n}+a_{1} R=I_{n+1}+a_{1} R$ and $z \in I_{n+1}$, then $z=w+a_{1} x$, where $w \in J I_{n}$ and $x \in R$. Thus $a_{1} x=z-w \in I_{n+1}$ so that $x \in\left(I_{n+1}: a_{1}\right)$. Since $a_{1}^{*} \in G(\mathfrak{F})$ is regular, we have $\left(I_{n+1}: a_{1}\right)=I_{n}$. Therefore $x \in I_{n}$ so that $z \in J I_{n}$. 
In [6], Cortadellas and Zarzuela show under some assumptions that the regularity of the fiber cone is equal to reduction number of a minimal reduction $J$ of $I$. We verify the same result for equimultiple good filtration.

Proposition 2.11. Let $(A, \mathfrak{m})$ be a Noetherian local ring and let $\mathfrak{F}$ an equimultiple good filtration. Denote $\ell=\operatorname{dim} F(\mathfrak{F})$ and assume grade $I_{1}=\ell$, $\operatorname{grade} G(\mathfrak{F})_{+} \geq \ell-1$ and $\operatorname{depth} F(\mathfrak{F}) \geq \ell-1$. Let $J \subseteq I_{1}$ be a minimal reduction of $\mathfrak{F}$ and denote $r=r_{J}(\mathfrak{F})$, the reduction number of $\mathfrak{F}$ with respect to $J$. Then we have the following:

(1) $\operatorname{reg} F(\mathfrak{F})=r$;

(2) $F(\mathfrak{F})$ is a Cohen-Macaulay ring if and only if

$$
\lambda\left(I_{n} /\left(\mathfrak{m} I_{n}+J I_{n-1}\right)\right)=\sum_{i=0}^{\ell}(-1)^{i}\left(\begin{array}{l}
\ell \\
i
\end{array}\right) \mu\left(I_{n-i}\right),
$$

for $1 \leq n \leq r(\mathfrak{F})$.

Proof. If $\ell=1$ the results follow by [23, Lemma 4.2] and Lemma 2.9. Suppose $\ell>1$. Since grade $I_{1}=\ell$ we may find a minimal reduction $J=$ $\left(a_{1}, \ldots, a_{\ell}\right)$ of $\mathfrak{F}$ such that $a_{1}, \ldots, a_{\ell} \in A$ a regular sequence, $a_{1}^{*}, \ldots, a_{l-1}^{*} \in$ $G(\mathfrak{F})$ is a regular sequence and $a_{1}^{o}, \ldots, a_{l-1}^{o} \in F(\mathfrak{F})$ is a regular sequence. As $I_{1} /\left(a_{1}, \ldots, a_{\ell-1}\right) \subset A /\left(a_{1}, \ldots, a_{\ell-1}\right)$ contains only one regular element and besides $\operatorname{dim} F\left(\mathfrak{F} /\left(a_{1}, \ldots, a_{\ell-1}\right)\right)=1$ (see [22, Proposition 2.5]) and $r_{J}(\mathfrak{F})=$ $r_{J / J_{\ell-1}}\left(\mathfrak{F} / J_{\ell-1}\right)$ (see above Lemma), we have that

$$
\operatorname{reg} F(\mathfrak{F})=\operatorname{reg} F(\mathfrak{F}) /\left(a_{1}^{o}, \ldots, a_{l-1}^{o}\right)=\operatorname{reg} F\left(\mathfrak{F} / J_{\ell-1}\right)=r .
$$

We have done (1).

By [1, Theorem 2.1.3] and the fact that

$$
F\left(\mathfrak{F} /\left(a_{1}, \ldots, a_{\ell-1}\right)\right) \cong F(\mathfrak{F}) /\left(a_{1}^{o}, \ldots, a_{l-1}^{o}\right),
$$

we have that $F(\mathfrak{F})$ is Cohen-Macaulay if and only if $F\left(\mathfrak{F} /\left(a_{1}, \ldots, a_{\ell-1}\right)\right)$ is Cohen-Macaulay. Let denote by $\bar{I}_{n}=\left(I_{n}+J_{\ell-1}\right) / J_{\ell-1}$ the $n$-th term of $\mathfrak{F} /\left(a_{1}, \ldots, a_{\ell-1}\right)$ and $\mathfrak{m}_{\ell-1}=\mathfrak{m} / J_{\ell-1} \subset A / J_{\ell-1}$. By Theorem 2.9, the argument above is equivalent to

$$
\lambda\left(\bar{I}_{n} / \mathfrak{m}_{\ell-1} \bar{I}_{n}+a_{\ell} \bar{I}_{n-1}\right)=\mu\left(\bar{I}_{n-1}\right)-\mu\left(\bar{I}_{n-1}\right),
$$

for $0 \leq n \leq r$. By the isomorphism theorem and [13, Proposition 3.5], we have

$$
\begin{aligned}
\bar{I}_{n} / \mathfrak{m}_{\ell-1} \bar{I}_{n}+a_{\ell} \bar{I}_{n-1} & \cong I_{n} /\left(\mathfrak{m} I_{n}+a_{l} I_{n-1}+I_{n} \cap\left(a_{1}, \ldots, a_{\ell-1}\right)\right) \\
& \cong I_{n} /\left(\mathfrak{m} I_{n}+\left(a_{1}, \ldots, a_{\ell}\right) I_{n-1}\right) .
\end{aligned}
$$

By Lemma 2.8, $\mu\left(\bar{I}_{n-1}\right)-\mu\left(\bar{I}_{n-1}\right)=\sum_{i=0}^{\ell}(-1)^{i}\left(\begin{array}{l}\ell \\ i\end{array}\right) \mu\left(I_{n-i}\right)$. Then (2) follows. 
Notation 2.12. By [22, Proposition 2.2], we get a minimal reduction $J=$ $\left(x_{1}, \ldots, x_{d}\right)$ of $\mathfrak{F}$ such that $x_{1}^{*}, \ldots, x_{d}^{*} \in G(\mathfrak{F})$ and $x_{1}^{o}, \ldots, x_{d}^{o} \in F(\mathfrak{F})$ are superficial sequence. Denote $J_{i}=\left(x_{1}, \ldots, x_{i}\right), J_{0}=0$ and $J^{*}=\left(x_{1}^{*}, \ldots, x_{d}^{*}\right)$, $J^{o}=\left(x_{1}^{o}, \ldots, x_{d}^{o}\right)$.

Lemma 2.13. Suppose $G(\mathfrak{F})$ and $F(\mathfrak{F})$ are Cohen-Macaulay rings. Then $G(\mathfrak{F})$ is Gorenstein if and only if $G\left(\mathfrak{F} / J_{i}\right)$ is Gorenstein and $F(\mathfrak{F})$ is Gorenstein if and only if $F\left(\mathfrak{F} / J_{i}\right)$ is Gorenstein.

Proof. Let $J=\left(x_{1}, \ldots, x_{d}\right)$ be a minimal reduction of $\mathfrak{F}$ such that $x_{1}^{*}, \ldots, x_{d}^{*} \in$ $G(\mathfrak{F})$ and $x_{1}^{o}, \ldots, x_{d}^{o} \in F(\mathfrak{F})$ are superficial sequence. Since $G(\mathfrak{F})$ and $F(\mathfrak{F})$ are Cohen-Macaulay rings, these sequences are also regular sequences ([13, Lemma 2.1]). Then for any $i$ such that $1 \leq i \leq d, G(\mathfrak{F}) /\left(x_{1}^{*}, \ldots, x_{i}^{*}\right) \cong G\left(\mathfrak{F} /\left(x_{1}, \ldots, x_{i}\right)\right)$ and $F(\mathfrak{F}) /\left(x_{1}^{*}, \ldots, x_{i}^{*}\right) \cong F\left(\mathfrak{F} /\left(x_{1}, \ldots, x_{i}\right)\right)$ (see [16, Lemma 3.4] and [23, Lemma 4.5]). By [1, Proposition 3.1.19], the result follows.

The next Theorem gives, for Hilbert filtration, a characterization for fiber cone to be Gorenstein ring. We make a change in the conditions involving the lengths used in [19].

Theorem 2.14. Let $(A, \mathfrak{m})$ be a Noetherian local ring and $J$ a minimal reduction of a Hilbert filtration $\mathfrak{F}$ with reduction number $r$. Suppose $G(\mathfrak{F})$ is Gorenstein and $F(\mathfrak{F})$ is Cohen-Macaulay. Therefore $F(\mathfrak{F})$ is Gorenstein if and only if

$$
\lambda\left(\frac{\left(\left(I_{n+1}+J\right): \mathfrak{m}\right) \cap I_{n}}{I_{n+1}+J I_{n-1}}\right)=\sum_{i=0}^{d}(-1)^{i}\left(\begin{array}{l}
d \\
i
\end{array}\right) \mu\left(I_{n-i}\right) \text { and } \lambda\left(\frac{\left(I_{1}: \mathfrak{m}\right)}{I_{1}}\right)=1
$$

for $1 \leq n \leq r$

Proof. Since $G(\mathfrak{F})$ and $F(\mathfrak{F})$ are Cohen-Macaulay, by [22, Proposition 2.2] and [13, Lemma 2.1], we get a minimal reduction $J$ generated by elements whose the corresponding images form a regular sequence in $G(\mathfrak{F})$ and $F(\mathfrak{F})$. By applying the Proposition 2.1 to $\mathfrak{F} / J$ and by using the fact of $\operatorname{dim} F(\mathfrak{F} / J)=0$, one can prove

$$
\omega_{F(\mathfrak{F} / J)}=\bigoplus_{n \in \mathbb{Z}}\left[\frac{\left(\left(I_{n+r+1}+J\right): \mathfrak{m}\right) \cap I_{n+r}+J}{I_{n+r+1}+J}\right] .
$$

By the isomorphism theorem and the fact that $J \cap I_{n}=J I_{n-1}$ for all $n$, we have

$$
\left[\omega_{F(\mathfrak{F} / J)}\right]_{n-r} \cong \frac{\left(\left(I_{n+1}+J\right): \mathfrak{m}\right) \cap I_{n}}{I_{n+1}+J I_{n-1}}
$$


If $F(\mathfrak{F})$ is Gorenstein, by Lemma 2.13 , it follows that $F(\mathfrak{F} / J)$ is also Gorenstein, and then $\omega_{F(\mathfrak{F} / J)}=F(\mathfrak{F} / J)(r)$. It is easy to check the equality $[F(\mathfrak{F} / J)(r)]_{n-r}=I_{n} /\left(\mathfrak{m} I_{n}+J I_{n-1}\right)$. Then by applying $\lambda$ at $(2.3)$ and at the last equality, it gives

$$
\lambda\left(\frac{\left(\left(I_{n+1}+J\right): \mathfrak{m}\right) \cap I_{n}}{I_{n+1}+J I_{n-1}}\right)=\lambda\left(\frac{I_{n}}{\mathfrak{m} I_{n}+J I_{n-1}}\right),
$$

for all $n$. By Proposition 2.11, we have the result desired.

For the converse, we again use the Proposition 2.11 to obtain that $\lambda\left(\left(\left(I_{n+1}+\right.\right.\right.$ $\left.\left.J): \mathfrak{m}) \cap I_{n}\right) /\left(I_{n+1}+J I_{n-1}\right)\right)=\lambda\left(I_{n} /\left(\mathfrak{m} I_{n}+J I_{n-1}\right)\right)$, for $0 \leq n \leq r$. Of course this equality is true for all $n$ since $J$ is a reduction of $\mathfrak{F}$. Hence by $(2.3), \lambda\left(\left[\omega_{F(\mathfrak{F} / J)}\right]_{n}\right)=\lambda\left([F(\mathfrak{F} / J)(r)]_{n}\right)$ for all $n$ and then $\lambda\left(\omega_{F(\mathfrak{F} / J)}\right)=$ $\lambda(F(\mathfrak{F} / J)(r))=\lambda(F(\mathfrak{F} / J))$. Let $\mu=\mu\left(\omega_{F(\mathfrak{F} / J)}\right)$ be the minimal number of generators of $\omega_{F(\mathfrak{F} / J)}$. We may construct a natural surjective map $\psi$ : $H \rightarrow \omega_{F(\mathfrak{F} / J)}$ such that $H$ is a free graded $F(\mathfrak{F} / J)$-module of rank $\mu$. By $\left[9\right.$, Corollary 36.11], $\omega_{F(\mathfrak{F} / J)}$ has finite injective dimension which is equal to $\operatorname{depth} F(\mathfrak{F} / J)=\operatorname{dim} F(\mathfrak{F} / J)=0$. This way, $\omega_{F(\mathfrak{F} / J)}$ is an injective module and consequently $\operatorname{Hom}_{F(\mathfrak{F} / J)}\left(-, \omega_{F(\mathfrak{F} / J)}\right)$ is an exact functor. Then we also have a surjective map

$$
\psi^{*}: \operatorname{Hom}_{F(\mathfrak{F} / J)}\left(\omega_{F(\mathfrak{F} / J)}, \omega_{F(\mathfrak{F} / J)}\right) \rightarrow \operatorname{Hom}_{F(\mathfrak{F} / J)}\left(H, \omega_{F(\mathfrak{F} / J)}\right) .
$$

By [2, Theorem 13.3.4(ii)],

$$
\operatorname{Hom}_{F(\mathfrak{F} / J)}\left(\omega_{F(\mathfrak{F} / J)}, \omega_{F(\mathfrak{F} / J)}\right) \cong F(\mathfrak{F} / J) .
$$

Naturally

$$
\operatorname{Hom}_{F(\mathfrak{F} / J)}\left(H, \omega_{F(\mathfrak{F} / J)}\right) \cong \bigoplus_{\mu} \omega_{F(\mathfrak{F} / J)}
$$

We have gotten a surjective map $F(\mathfrak{F} / J) \rightarrow \bigoplus_{\mu} \omega_{F(\mathfrak{F} / J)}$. Hence

$$
\mu \cdot \lambda\left(\omega_{F(\mathfrak{F} / J)}\right)=\lambda\left(\bigoplus_{\mu} \omega_{F(\mathfrak{F} / J)}\right) \leq \lambda(F(\mathfrak{F} / J)) .
$$

This implies that $\mu=1$. By [1, Proposition 3.6.11], $F(\mathfrak{F} / J)$ is Gorenstein, and then by Lemma 2.13 the result follows.

Corollary 2.15. Let $(A, \mathfrak{m})$ be a Noetherian local ring and $J$ a minimal reduction of a Hilbert filtration $\mathfrak{F}$. Suppose $G(\mathfrak{F})$ is Gorenstein and $F(\mathfrak{F})$ is Cohen-Macaulay. Then $F(\mathfrak{F})$ is Gorenstein if and only if

$$
\mu\left(I_{n}\right)=\lambda\left(\frac{\left(\left(I_{n+1}+J\right): \mathfrak{m}\right) \cap I_{n}}{I_{n+1}+J \mathfrak{m} I_{n-1}}\right)
$$

for $0 \leq n \leq r$ 
Proof. By Theorem 2.14, we have

$$
\lambda\left(\frac{\left(\left(I_{n+1}+J\right): \mathfrak{m}\right) \cap I_{n}}{I_{n+1}+J I_{n-1}}\right)=\lambda\left(\frac{I_{n}}{\mathfrak{m} I_{n}+J I_{n-1}}\right)
$$

for all $n$. Since

$$
\lambda\left(\frac{\left(\left(I_{n+1}+J\right): \mathfrak{m}\right) \cap I_{n}}{I_{n+1}+J I_{n-1}}\right)=\lambda\left(\frac{I_{n}}{I_{n+1}+J I_{n-1}}\right)-\lambda\left(\frac{I_{n}}{\left(\left(I_{n+1}+J\right): \mathfrak{m}\right) \cap I_{n}}\right)
$$

and

$$
\lambda\left(\frac{I_{n}}{I_{n+1}+J I_{n-1}}\right)=\lambda\left(\frac{I_{n}}{\mathfrak{m} I_{n}+J I_{n-1}}\right)+\lambda\left(\frac{\mathfrak{m} I_{n}+J I_{n-1}}{I_{n+1}+J I_{n-1}}\right),
$$

one can obtain

$$
\lambda\left(\frac{\mathfrak{m} I_{n}}{I_{n+1}+J \mathfrak{m} I_{n-1}}\right)=\lambda\left(\frac{\mathfrak{m} I_{n}+J I_{n-1}}{I_{n+1}+J I_{n-1}}\right)=\lambda\left(\frac{I_{n}}{\left(\left(I_{n+1}+J\right): \mathfrak{m}\right) \cap I_{n}}\right) .
$$

But

$$
\lambda\left(\frac{\mathfrak{m} I_{n}}{I_{n+1}+J \mathfrak{m} I_{n-1}}\right)=\lambda\left(\frac{I_{n}}{I_{n+1}+J \mathfrak{m} I_{n-1}}\right)-\lambda\left(\frac{I_{n}}{\mathfrak{m} I_{n}}\right)
$$

so that

$$
\lambda\left(\frac{I_{n}}{\mathfrak{m} I_{n}}\right)=\lambda\left(\frac{\left(\left(I_{n+1}+J\right): \mathfrak{m}\right) \cap I_{n}}{I_{n+1}+J \mathfrak{m} I_{n-1}}\right) .
$$

For the converse, firstly note that

$$
\lambda\left(\frac{I_{n}}{\mathfrak{m} I_{n}}\right)=\lambda\left(\frac{\mathfrak{m} I_{n}+J I_{n-1}}{\mathfrak{m} I_{n}}\right)+\lambda\left(\frac{I_{n}}{\mathfrak{m} I_{n}+J I_{n-1}}\right)
$$

and

$\lambda\left(\frac{\left(\left(I_{n+1}+J\right): \mathfrak{m}\right) \cap I_{n}}{I_{n+1}+J \mathfrak{m} I_{n-1}}\right)=\lambda\left(\frac{I_{n+1}+J I_{n-1}}{I_{n+1}+J \mathfrak{m} I_{n-1}}\right)+\lambda\left(\frac{\left(\left(I_{n+1}+J\right): \mathfrak{m}\right) \cap I_{n}}{I_{n+1}+J I_{n-1}}\right)$.

By [4, Proposition 3.7], one can conclude that

$$
\lambda\left(\frac{\mathfrak{m} I_{n}+J I_{n-1}}{\mathfrak{m} I_{n}}\right)=\lambda\left(\frac{I_{n+1}+J I_{n-1}}{I_{n+1}+J \mathfrak{m} I_{n-1}}\right) .
$$

In [1, Theorem 4.5.7], it is proved that if $G(\mathfrak{F})$ is Gorenstein, so is $A$. But in general, it does not occur for fiber cone: In [20, Example 6.3], it is shown that if $A=k\left[\left[t^{4}, t^{5}, t^{6}, t^{7}\right]\right], I=\left(t^{4}, t^{5}, t^{6}\right)$ and $J=\left(t^{4}\right)$, then $F(I)$ is Gorenstein.

In the Corollary 2.16, we see that if $\mathfrak{F}$ is a Hilbert filtration and both $G(\mathfrak{F})$ and $F(\mathfrak{F})$ are Gorenstein, then $A / I_{1}$ is Gorenstein. 
Corollary 2.16. Let $(A, \mathfrak{m})$ be a Noetherian local ring and $\mathfrak{F}$ a Hilbert filtration. If both $G(\mathfrak{F})$ and $F(\mathfrak{F})$ are Gorenstein, then so is $A / I_{1}$.

Proof. By Theorem 2.14, for $n=0$, we have $\lambda\left(\left(I_{1}: \mathfrak{m}\right) / I_{1}\right)=\lambda(A / \mathfrak{m})=1$. It implies that $\left(I_{1}: \mathfrak{m}\right) / I_{1} \cong A / \mathfrak{m}$. By [18, Theorem 11.12], the result is done.

In the article [20], the authors studied the Gorenstein property of fiber cones of ideals of (almost) minimal multiplicity and Sally ideals. Now we will use the Theorem 2.14 to obtain more results in these cases.

Let $I$ be an $\mathfrak{m}$-primary ideal of a Cohen-Macaulay local ring. We recall that $I$ is said to be a Sally ideal if it satisfies the condition $\lambda\left(I^{2} / J I\right)=1$, for any minimal reduction $J$ of the ideal $I$. The ideal $I$ is said to be of minimal multiplicity (resp. of almost minimal multiplicity) if it is satisfies the equality $\mathfrak{m} I=\mathfrak{m} J(\operatorname{resp} . \lambda(\mathfrak{m} I / \mathfrak{m} J)=1)$.

Corollary 2.17. Suppose $G(I)$ is Gorenstein and $F(I)$ is Cohen-Macaulay. If $I$ is either a Sally ideal or an ideal of almost minimal multiplicity then $F(I)$ is Gorenstein if and only if

$$
\lambda\left(\frac{\left(\left(I^{2}+J\right): \mathfrak{m}\right) \cap I}{I^{2}+J}\right)=\lambda\left(\frac{I}{\mathfrak{m} I+J}\right) \text { and } R / I \text { is Gorenstein. }
$$

Proof. If $I$ is a Sally ideal, we have $\mathfrak{m} I^{n} \subseteq J I^{n-1}$ for $n \geq 2$. If $I$ is an ideal of almost minimal multiplicity then $\mathfrak{m} I^{2} \subseteq J \mathfrak{m}$. In both cases the equalities of lengths in Theorem 2.14 are automatically satisfied for $n \geq 2$.

Corollary 2.18. Suppose $G(I)$ is Gorenstein and $F(I)$ is Cohen-Macaulay. If $I$ is an ideal of minimal multiplicity then $F(I)$ is Gorenstein if and only if $R / I$ is Gorenstein.

Proof. Since $\mathfrak{m} I=\mathfrak{m} J$, the result follows from Theorem 2.14.

Since $\omega_{F(\mathfrak{F})}$ is a $F(\mathfrak{F})$-module, we may ask if it is also an ideal of $F(\mathfrak{F})$. Under certain assumptions, this result may be obtained.

Corollary 2.19. Let $(A, \mathfrak{m})$ be a Noetherian local ring and $\mathfrak{F}$ a Hilbert filtration. Assume that $G(\mathfrak{F})$ is Gorenstein, $F(\mathfrak{F})$ is Cohen-Macaulay and $A / I_{1}$ is Gorenstein. If we suppose that $\left(I_{n+r-d+1}: \mathfrak{m}\right) \cap \mathfrak{m} I_{n+r-d}=I_{n+r-d+1}$ for all $n \geq d-r+1, \omega_{F(\mathfrak{F})}$ is a submodule of $F(\mathfrak{F})$.

Proof. By hypothesis, $I_{n+1} \subseteq \mathfrak{m} I_{n}$ for any $n$. Then there is a natural map

$$
\psi_{n}: \frac{\left(I_{n+r-d+1}: \mathfrak{m}\right) \cap I_{n+r-d}}{I_{n+r-d+1}} \rightarrow \frac{I_{n+r-d}}{\mathfrak{m} I_{n+r-d}}
$$


for $n \geq d-r$, where

$$
\operatorname{Ker} \psi_{n}=\frac{\left(I_{n+r-d+1}: \mathfrak{m}\right) \cap \mathfrak{m} I_{n+r-d}}{I_{n+r-d+1}} .
$$

By (2.2), it gives a natural $F(\mathfrak{F})$-linear map

$$
\psi: \omega_{F(\mathfrak{F})} \rightarrow F(\mathfrak{F}) .
$$

Since $A / I_{1}$ is Gorenstein, by using [18, Theorem $\left.11.12(3)\right], \operatorname{Soc}\left(A / I_{1}\right)=\left(I_{1}\right.$ : $\mathfrak{m}) / I_{1} \cong A / \mathfrak{m}$, which gives Ker $\psi_{d-r}=0$. Then by last hypothesis,

$$
\operatorname{ker} \psi=\bigoplus_{n \geq d-r} \frac{\left(I_{n+r-d+1}: \mathfrak{m}\right) \cap \mathfrak{m} I_{n+r-d}}{I_{n+r-d+1}}=0 .
$$

The result is concluded.

In [19], it is showed that $e_{0}\left(\omega_{F(\mathfrak{F})}\right)<e_{0}\left(\omega_{G(\mathfrak{F})}\right)$ unless $I=\mathfrak{m}$. For Hilbert filtration the result is similar.

Proposition 2.20. Let $(A, \mathfrak{m})$ be a Noetherian local ring and $\mathfrak{F}$ a Hilbert filtration such that $G(\mathfrak{F})$ and $F(\mathfrak{F})$ are Cohen-Macaulay. Then

$$
e_{0}\left(\omega_{F(\mathfrak{F})}\right) \leq e_{0}\left(\omega_{G(\mathfrak{F})}\right) .
$$

In addition, if $G(\mathfrak{F})$ is Gorenstein, we have the equality if and only if $I_{1}=\mathfrak{m}$.

Proof. Proposition 2.1 gives that $\omega_{F(\mathfrak{F})} \subseteq \omega_{G(\mathfrak{F})}$. By additivity of $e_{0}$,

$$
e_{0}\left(\omega_{F(\mathfrak{F})}\right) \leq e_{0}\left(\omega_{G(\mathfrak{F})}\right) \text {. }
$$

By hypothesis and the properties of canonical module we may find a minimal reduction $J=x_{1}, \ldots, x_{d}$ of $\mathfrak{F}$ such that $x_{1}^{*}, \ldots, x_{d}^{*} \in G(\mathfrak{F})$ are regular sequence and $\omega_{G(\mathfrak{F})}$-regular sequence and $x_{1}^{o}, \ldots, x_{d}^{o} \in F(\mathfrak{F})$ are regular sequence and $\omega_{F(\mathfrak{F})}$-regular sequence. By [16, Remark 4.1.11] and using the fact that $\operatorname{dim} F(\mathfrak{F})=0$ and $\operatorname{dim} G(\mathfrak{F})=0$, we have

$$
e_{0}\left(\omega_{G(\mathfrak{F})}\right)=e_{0}\left(\omega_{G(\mathfrak{F} / J)}\right)=\lambda(G(\mathfrak{F} / J))
$$

and

$$
e_{0}\left(\omega_{F(\mathfrak{F})}\right)=e_{0}\left(\omega_{F(\mathfrak{F} / J)}\right)=\lambda(F(\mathfrak{F} / J)) .
$$

If $e_{0}\left(\omega_{G(\mathfrak{F})}\right)=e_{0}\left(\omega_{F(\mathfrak{F})}\right)$, then $\lambda\left(\omega_{F(\mathfrak{F} / J)}\right)=\lambda\left(\omega_{G(\mathfrak{F} / J)}\right)$. By Proposition 2.1 , we have $\omega_{F(\mathfrak{F} / J)} \subseteq \omega_{G(\mathfrak{F} / J)}$, and hence $\omega_{F(\mathfrak{F} / J)}=\omega_{G(\mathfrak{F} / J)}$. Since $G(\mathfrak{F} / J)$ is Gorenstein and by using the isomorphism (2.3), we get $\left(I_{1}: \mathfrak{m}\right) / I_{1}=A / I_{1}$ which means $I_{1}=\mathfrak{m}$.

The converse follows trivially, since from assumption $I_{n+1} \subseteq \mathfrak{m} I_{n}$, it is easy to conclude that in this case $\mathfrak{F}=\left(\mathfrak{m}^{n}\right)_{n \geq 0}$.

Acknowlegment. We would like to thank the professors J. V. Jayanthan and $R$. Nanduri for the clarifications. 


\section{References}

[1] W. Bruns, J. Herzog, Cohen-Macaulay rings, Revised Edition, Cambridge Studies in Advanced Mathematics, 39. Cambridge University Press, Cambridge, 1998.

[2] M. P. Brodmann, R. Y. Sharp, Local cohomology: an algebraic introduction with geometric applications, Cambridge Studies in Advanced Mathematics, 60. Cambridge University Press, Cambridge, 1998.

[3] T. Cortadellas, Fiber cones with almost maximal depth, Comm. Algebra, 33, no. 3, 953-963, 2005.

[4] T. Cortadellas, S. Zarzuela, On the depth of the fiber cone of filtrations, J. Algebra 198, 1997, no 2, 428-445.

[5] T. Cortadellas, S. Zarzuela, Depth formulas for certain graded modules associated to a filtration: a survey, Geometric and combinatorial aspects of commutative algebra (Messina, 1999), 145-157, Lecture Notes in Pure and Appl. Math., 217, Dekker, New york, 2001.

[6] T.Cortadellas, S. Zarzuela, On the scruture of the fiber cone of ideals with analytic spread one, J. Algebra 317, 2007, no. 2, 759-785.

[7] S. Goto, K. Nishida, The Cohen-Macaulay and Gorenstein Rees algebras associated to filtrations, Mem. Amer. Math. Soc. 110, 1994, no. $22,5635-5656$.

[8] Gert-Martin Greuel, G. Pfister, A Singular introduction to commutative algebra, Second extended edition, Springer, Berlin, 2008.

[9] M. Herrmann; S. Ikeda, U. Orbanz, Equimultiplicity and blowing up, An algebraic study. With an appendix by B. Moonen. SpringerVerlag, Berlin, 1988.

[10] W. J. Heinzer, M-K. Kim, Properties of the fiber cone of ideals in local rings, Comm. Algebra 31 (7), 2003, 3529-3546.

[11] W. Heinzer, M. Kim, B. Ulrich, The Gorenstein and complete intersection properties of associated graded rings, J. Pure Appl. Algebra 201, 2005, no. 1-3, 264283.

[12] W. Heinzer, M. Kim, B. Ulrich, The Cohen-Macaulay and Gorenstein Properties of Rings Associated to Filtrations, Volume 39, Issue 10, 2011. 
[13] S. Huckaba, T. Marley, Hilbert coefficients and the depths of associated graded rings, J. London Math. Soc. (2) 56 , 1997, no. 1, 6476.

[14] M. Herrmann, J. Ribbe, S. Zarzuela, On the Gorenstein property of Rees and form rings of power of ideals, Trans. Amer. Math. Soc. 342, 1994, no. 2, 631643 .

[15] E. Hyry, On the Gorenstein property of the associated graded ring of power of a an ideal, Manuscripta Math. 80, 1993, 13-20.

[16] L. T. Hoa and S. Zarzuela, Reduction number and a-invariant of good filtrations, Comm. Algebra 14, 1994, no. 22, 5635-5656.

[17] S. Ikeda, On the Gorensteinness of Rees Algebras over local rings, Nagoya Math. J. 102, 135-154, 1986.

[18] S. Iyengar, G. Leuschke, A. Leykin, C. Miller, E. Miller, A. Singh, U. Walther, Twenty-four hours of local cohomology, Graduate Studies in Mathematics, 87. American Mathematical Society, Providence, RI, 2007.

[19] A. V. Jayanthan, R. Nanduri, Castelnuovo-Mumford regularity and gorensteinness of fiber cone, arXiv:1103.3555v1, 2011.

[20] A. V. Jayanthan, T. J. Puthenpurakal, J. K. Verma, On fiber cones of m-primary ideals, Canad. J. Math. 59, 2007, no 1, 109-126.

[21] M. Johnson and B. Ulrich, Artin-Nagata properties and CohenMacaulay associated graded rings, Compositio Math. 108, 1996, 7-29.

[22] A. V. Jayanthan, J. K. Verma, Hilbert coefficients and depth of fiber cones, Journal of Pure and Applied Algebra 201, 2005, 97-115.

[23] P. H. Lima, V. H. Pérez, Castelnuovo-Mumford regularity of the fiber Cone for good filtrations, Institute of Mathematics and Computer Science, University of São Paulo, 2011.

[24] D. G. Northcott and D. Rees, Reductions of ideals in local rings, Proc. Cambridge Philos Soc. 50, 1954, 145-158.

[25] N. V. Trung, The Castelnuovo regularity of the Rees algebra and the associated graded ring, Trans. of Amer. Math. Soc. 350, 1998, no. 7, 2813-2832.

[26] N. V. Trung, D. Q. Viêt, S. Zarzuela, When is the Rees algebra Gorenstein ?, J. Algebra 175, 1995, 137-156.

Received: December 11, 2013 